\title{
A Generalization of Kotzig's Theorem and its Application
}

\author{
Richard Cole* $\quad$ Łukasz Kowalik ${ }^{\dagger} \quad$ Riste Škrekovski ${ }^{\ddagger}$
}

\begin{abstract}
An edge of a graph is light when the sum of the degrees of its endvertices is at most 13. The well-known Kotzig Theorem states that every 3-connected planar graph contains a light edge. Later, Borodin [1] extended this result to the class of planar graphs of minimum degree at least 3 .

We deal with generalizations of these results for planar graphs of minimum degree 2. Borodin, Kostochka and Woodall [3] showed that each such graph contains a light edge or a member of two infinite sets of configurations, called 2-alternating cycles and 3 -alternators. This implies that planar graphs with maximum degree $\Delta \geq 12$ are $\Delta$ edge-choosable. We prove a similar result with 2-alternating cycles and 3-alternators replaced by five fixed bounded-sized configurations called crowns. This gives another proof of $\Delta$-edge-choosability of planar graphs with $\Delta \geq 12$. However, we show efficient choosability, i.e. we describe a linear-time algorithm for $\max \{\Delta, 12\}$-edgelist-coloring planar graphs. This extends the result of Chrobak and Yung [5].
\end{abstract}

\section{Introduction}

One of the best-known facts concerning planar graphs states that every planar graph contains a vertex of degree at most 5. Let the weight of edge $e=u v$, denoted by $w(e)$, be the sum of the degrees of its end-vertices, i.e. $w(e)=\operatorname{deg}_{G}(u)+\operatorname{deg}_{G}(v)$. We say that an edge is light when its weight is at most 13. In 1955 Kotzig [12] showed the following theorem.

Theorem 1.1 (Kotzig). Every 3-connected planar graph contains a light edge.

${ }^{*}$ Computer Science Department, New York University, 251 Mercer Street, New York, NY 10012, USA. cole@cs.nyu.edu. This paper is based in part upon work supported by the National Science Foundation under grants CCR 0105678 and CCF 0515127.

†Institute of Informatics, Warsaw University, Banacha 2, 02-097 Warsaw, Poland and Max-PlanckInstitute für Informatik, Stuhlsatzenhausweg 85, 66123 Saarbrücken, Germany. kowalik@mimuw.edu.pl. Supported in part by KBN grant 4T11C04425.

${ }_{\ddagger}^{\ddagger}$ Department of Mathematics, University of Ljubljana, Jedranska 19, 1111 Ljubljana, Slovenia. skreko@fmf.uni-lj.si. Supported in part by Ministry of Science and Technology of Slovenia, Research Program P1-0297. 
This result was an inspiration for dozens of papers, which form the now so-called light graph theory (see the surveys by Jendrol' and Voss [10,11] and the introduction in [13]).

Kotzig's theorem was generalized in several directions, see e.g. [2, 7, 15]. In particular, Erdös conjectured that it is valid also for planar graphs with vertices of degree at least 3 , and this was proved by Borodin [1]:

Theorem 1.2 (Borodin). Every simple planar graph with minimum degree $\delta \geq 3$ contains a light edge.

A light edge is not always present if the graph under consideration has vertices of degree 2; for example, consider the bipartite complete graph $K_{2, k}$ for any $k \geq 12$. In this example each vertex of degree $d \geq 12$ has many 2-neighbors. However, one can guarantee the existence of a light edge by bounding the number of 2-neighbors.

Proposition 1.3. Let $G$ be a simple planar graph with minimum degree $\delta \geq 2$ such that each d-vertex, $d \geq 12$, has at most $d-11$ neighbors of degree 2 . Then $G$ contains a light edge.

Proof. We may assume that every 2-vertex of $G$ is adjacent to two vertices of degree at least 12 for otherwise there is a light edge in $G$. Consider the graph $G^{\prime}$ obtained from $G$ by replacing each path $u x w$ such that $\operatorname{deg}(x)=2$ by an edge joining $u$ and $w$. Additionally we replace multiple edges by single ones. Clearly $G^{\prime}$ is a simple planar graph with vertices of degree at least 3 and by Theorem 1.2, $G^{\prime}$ contains an edge of weight at most 13 . Consider such an edge $u w$.

First assume that $u$ has a 2-neighbor $x$ in $G$. Then $\operatorname{deg}_{G}(u) \geq 12$ and in $G$ vertex $u$ has at least 11 neighbors of degree at least 3 which implies that $\operatorname{deg}_{G^{\prime}}(u) \geq 11$ and hence $u w$ has weight at least 14 , a contradiction.

Hence we may assume that $u$ has no 2-neighbor in $G$ and the same holds for $w$. It follows that $u w$ belongs to $G$. Also, $\operatorname{deg}_{G}(u)=\operatorname{deg}_{G^{\prime}}(u)$ and $\operatorname{deg}_{G}(w)=\operatorname{deg}_{G^{\prime}}(w)$, and hence $u w$ has in $G$ the same weight as in $G^{\prime}$.

Borodin, Kostochka, and Woodall [3] proved the following result, where the number of 2-neighbors is not bounded:

Theorem 1.4 (Borodin, Kostochka, and Woodall). Every planar graph with minimum degree $\delta \geq 2$ contains a light edge, a 2-alternating cycle or a 3-alternator.

In the above theorem a 2-alternating cycle is an even length cycle with every second vertex of degree 2, while a 3-alternator is a bipartite subgraph $F$ with partite sets $U, W$ such that, for each $u \in U, 2 \leq \operatorname{deg}_{F}(u)=\operatorname{deg}_{G}(u) \leq 3$, and for each $w \in W$, either $\operatorname{deg}_{F}(w) \geq 3$ or $w$ has exactly two neighbors in $U$, both of degree $14-\operatorname{deg}_{G}(w)$ (the latter case is possible only if $\operatorname{deg}_{G}(w)=11$ or 12 ).

In this paper, we give a similar result involving only five small fixed subgraphs, called crowns (see Section 2 for the definition and see Fig. 1 for an illustration), instead of 2alternating cycles and 3-alternators. 
Theorem 1.5. Every planar graph with minimum degree $\delta \geq 2$ contains a light edge or a $k$-crown, for some $k \in\{1, \ldots, 5\}$.

Unlike 2-alternating cycles and 3-alternators the five crowns have bounded size and are contained in the "neighborhood" of one vertex.

\subsection{Applications}

Let $G$ be a graph. An edge-list assignment $L: E(G) \rightarrow \mathcal{P}(\boldsymbol{N})$ is a function that assigns to each edge $e$ of $G$ a set (or a list) $L(e)$ of admissible colors. A function $\lambda: E(G) \rightarrow \boldsymbol{N}$ is an $L$-edge-coloring if $\lambda(e) \in L(e)$ for every $e \in E(G)$, and $\lambda(e) \neq \lambda(f)$ for every pair of incident edges $e, f \in E(G)$. If $G$ admits an $L$-edge-coloring, it is $L$-edge-colorable. For $k \in \boldsymbol{N}$, a graph $G$ is $k$-edge-choosable if it has an $L$-edge-coloring for every edge-list assignment $L$ such that $|L(e)| \geq k$ for each $e \in E(G)$.

Throughout the paper $\Delta(G)$ will denote the maximum degree of graph $G$, i.e., the largest of the vertex degrees in $G$. Usually it is clear which graph we refer to and then we simply write $\Delta$.

Although it is conjectured that if a graph is $k$-edge-colorable then it is also $k$-edgechoosable, there is no analog of Vizing's Theorem for list-coloring, i.e. it is not known whether every graph is $\Delta+\mathcal{O}(1)$-choosable. However Borodin, Kostochka and Woodall [3] showed the following theorem:

Theorem 1.6 (Borodin, Kostochka, and Woodall). Every planar graph with maximum degree $\Delta \geq 12$ is $\Delta$-edge-choosable.

A subgraph of a planar graph is reducible when it cannot appear in a minimal counterexample for Theorem 1.6. In this sense, a light edge is reducible (see the paragraph "Edges of Bounded Weight" below). In Section 3 we show that crowns are reducible. Together with our main result this gives a new proof of Theorem 1.6.

We also consider efficient algorithms for edge-list-coloring planar graphs. Then given an $n$-vertex graph $G$ and an edge-list assignment $L$ such that lists have length $\max \{\Delta, 12\}$, one has to compute an $L$-edge-coloring of $G$. Note that the size of the input is $\Theta(|E(G)| \Delta)$, which is bounded by $\mathcal{O}(n \Delta)$ when $G$ is planar. Hence $\mathcal{O}(n \Delta)$-time algorithms are considered to be linear. Additionally, we assume that each list of admissible colors is sorted. If this assumption is not met the lists can be bucket-sorted in $\mathcal{O}(|E(G)| \Delta+M)$ time, where $M$ denotes the value of the largest color in the lists. Hence, equivalently one can assume that $M=\mathcal{O}(|E(G)| \Delta)$, which seems to be very natural. We will refer to it as the small colors assumption.

The proof of the 2-choosability criterion by Erdős, Rubin and Taylor [8] (proved earlier by Vizing [14]) yields a linear-time algorithm for optimally edge-list-coloring graphs with $\Delta=2$. For $\Delta=3$ there is a linear-time algorithm for 4-edge-list-coloring general graphs due to Gabow and Skulrattanakulchai [9]. For higher values of $\Delta$ one can use simple algorithms which rely on the existence in a planar graph of an edge of low weight. 
Edges of Bounded Weight. Assume we want to edge-list-color a planar graph $G$ with maximum degree $\Delta$ and with lists of length at least $D$. When an algorithm finds in $G$ an edge $e$ of weight at most $D+1$ then this edge is removed and the resulting graph is colored recursively. Since there are at most $D-1$ edges incident with $e$, these edges do not use all colors from list $L(e)$ and we can color $e$ with one of the remaining colors. Observe that this proves that light edges are reducible. Also note that when $\Delta=\mathcal{O}(1)$ this algorithm has linear time complexity. When $\Delta$ is not bounded, but the small color assumption holds, the algorithm can be also implemented to work in linear time (see Lemma 4.1). Clearly, any graph can be edge-list-colored from lists of length $D=2 \Delta-1$, since then any edge has weight at most $D+1$. For $\Delta=4,5$ nothing better is known, even for planar graphs; just note that for these values of $\Delta$, there are planar graphs with all edges of weight $2 \Delta$, for example, consider the octahedron and the dodecahedron. For $\Delta=6, \ldots, 10$ we can use the result of Borodin [1]: every planar graph of minimum degree at least 4 contains an edge of weight at most 11 . Hence any planar graph contains an edge of weight at most $\max \{\Delta+3,11\}$ and can be edge-list-colored in linear time from lists of length $\max \{\Delta+2,10\}$. For $\Delta \geq 11$ we can take advantage of Theorem 1.2. As before, it immediately yields a linear-time algorithm which requires lists containing $\max \{\Delta+1,12\}$ colors.

Ordinary Edge-Coloring. Chrobak and Yung [5] presented a linear-time algorithm for $\max \{\Delta, 19\}$-edge-coloring planar graphs. Although it was not mentioned explicitly their algorithm can be easily adapted to the list version of the problem. Then its time complexity increases to $\mathcal{O}(n \Delta)$, provided that the small colors assumption holds. There is also an $\mathcal{O}(n \log n)$-time algorithm due to Chrobak and Nishizeki [4] and a very recent $\mathcal{O}(n)$-time algorithm by Cole and Kowalik [6], both for $\max \{\Delta, 9\}$-edge-coloring planar graphs. However, as far as we know neither of these two algorithms can be extended to the edge-list-coloring problem.

Our Algorithm. We show an $\mathcal{O}(\Delta n)$-time algorithm for $\max \{\Delta, 12\}$-list-coloring planar graphs. The algorithm does not require a plane embedding of the input graph. This extends the algorithm of Chrobak and Yung [5].

\section{The Main Result}

In this section we present the main result of the paper, i.e. a generalization of Kotzig's theorem.

Definition 1. Let $G$ be a multigraph, and let $S$ be a subgraph of $G$, whose vertices are $v, x_{1}, x_{2}, \ldots, x_{2 k+1}$, for some $k \geq 1$. We call $S$ a crown of size $k$ around $v$ (shortly, a $k$-crown or just a crown), if the following conditions are satisfied:

(i) $E(S)=\left\{v x_{2 i+1}: i=0, \ldots, k\right\} \cup\left\{x_{i} x_{i+1}: i=1, \ldots, 2 k\right\}$, 


\begin{tabular}{|c|c|c|l|}
\hline$\Delta$ & length of lists & time & \multicolumn{1}{|c|}{ paper } \\
\hline \hline 2 & optimal & $\mathcal{O}(n)$ & Vizing [14]; Erdős, Rubin and Taylor [8] \\
\hline 3 & $\Delta+1$ & $\mathcal{O}(n)$ & Gabow, Skulrattanakulchai [9] \\
\hline 4,5 & $2 \Delta-1$ & $\mathcal{O}(n)$ & folklore \\
\hline 6,7 & 10 & $\mathcal{O}(n)$ & Borodin [1] \\
\hline $8,9,10$ & $\Delta+2$ & $\mathcal{O}(n)$ & Borodin [1] \\
\hline 11 & $\Delta+1$ & $\mathcal{O}(n)$ & Borodin [1] \\
\hline$\geq 12$ & $\Delta$ & $\mathcal{O}(\Delta n)$ & this work \\
\hline \multicolumn{3}{|r}{} \\
\end{tabular}

Table 1: Linear-time algorithms for list-edge-coloring planar graphs. For $\Delta=4,5, \ldots, 11$ the algorithms consist of finding a reducible edge whose existence is obvious or guaranteed by the cited paper.

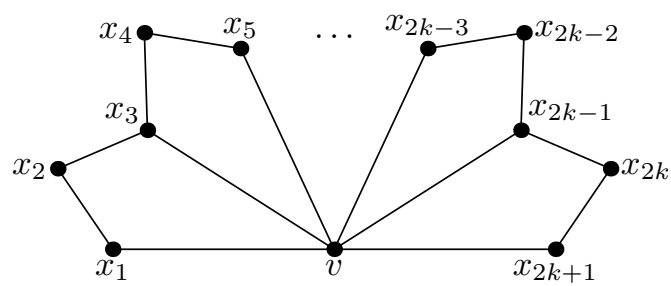

Figure 1: A $k$-crown.

(ii) $\operatorname{deg}_{G}\left(x_{1}\right)=\operatorname{deg}_{G}\left(x_{2 k+1}\right)=2$,

(iii) for each $i=1,2, \ldots, k-1, \operatorname{deg}_{G}\left(x_{2 i+1}\right)=3$, and

(iv) vertices $v, x_{1}, x_{2}, \ldots, x_{2 k+1}$ are all distinct.

Moreover, a crown of size at most 5 will be called a small crown.

Observe that a crown $S$ is not necessarily an induced subgraph of $G$. Thus, for an example, $G$ may have edges $v x_{2}$ or $x_{2} x_{4}$ which are not in $S$. We note here that every edge of a crown $S$ in a graph $G$ has an end-vertex of degree 2 or 3 in $G$. Thus, if in $G$ one connects two vertices of degree $\geq 3$ by an additional edge, then a new crown is not introduced. These remarks will be used later in some arguments. Now we are ready to prove the main result of the paper.

Proof of Theorem 1.5. Clearly, it suffices to prove the result for connected graphs. In this proof we identify planar graphs with their fixed plane embeddings. This allows us to consider faces of these graphs. The length of a face $f$, denoted by $\ell(f)$, is the length of the shortest closed walk induced by all edges incident with $f$. In order to make the proof easier, we will allow multiple edges and loops in our graphs (where each loop contributes 2 to the degree of its endvertex) with the following restrictions: 
(a) each face of $G$ is of length $\geq 3$;

(b) for each 2-vertex, at least one of the faces incident with it is not a triangle, and the two edges incident with it are not parallel.

Clearly, every simple planar graph, except $C_{3}$, satisfies these conditions. However, for $C_{3}$ the theorem holds trivially.

Suppose that $G$ is a counterexample of the theorem on $|V(G)|$ vertices with the maximum possible number of edges. Let $G^{*}$ be the graph obtained from $G$ by removing all its 2-vertices.

\section{Claim 1. $G^{*}$ is a triangulation.}

The proof is by contradiction. We will show that if $G^{*}$ is not a triangulation then $G$ is not maximal, i.e., that one can add an edge to $G$ so that it is still a counterexample for the theorem.

First assume that $G^{*}$ is disconnected. Then there is a 2-vertex $x \in V(G)$ with neighbors $u$ and $v$, each of degree $\geq 12$, such that $u$ and $v$ belong to different components of $G^{*}$. Consider the graph $G \cup\{u v\}$ such that the added edge $u v$ is embedded in a face of $G$ containing $u$ and $v$. Clearly it is a plane multigraph with neither light edges nor crowns. Note that $u$ and $v$ are not adjacent in $G$ for otherwise they are also adjacent in $G^{*}$. Hence $G \cup\{u v\}$ satisfies conditions $(a)$ and $(b)$. This contradicts maximality of $G$ and so $G^{*}$ is connected. Now it remains to show that every face of $G^{*}$ is of length 3.

Graph $G^{*}$ does not contain a face of length 1 for otherwise $G$ contains a 2-vertex incident with parallel edges. If $G^{*}$ contains a 2-face $f=x y x$ it implies that $f$ contains at least one 2-vertex of graph $G$. If $f$ contains precisely one 2-vertex then $G$ violates $(b)$, a contradiction. If $f$ contains at least two 2-vertices, $G$ contains two adjacent 2 -vertices or a 1-crown, a contradiction again. Hence each face of $G^{*}$ has length at least 3 .

Suppose that $f$ is a face of $G^{*}$ of length $k \geq 4$. Since $G^{*}$ is connected, $f$ has a facial walk, i.e. the shortest walk consisting of edges incident with $f$. Let $x_{0} x_{1} \ldots x_{x-1} x_{k}$ be the vertices of this walk in clockwise order, $x_{0}=x_{k}$.

We first prove that if $f$ contains a 2-vertex from $G$, say $w$, and $x_{i}, x_{j}$ denote the neighbors of $w$, then $i=j \pm 1(\bmod k)$. Otherwise, consider the graph $G^{\prime}$ obtained from $G$ by connecting $x_{i}$ and $x_{j}$ by a new edge $x_{i} x_{j}$. Obviously, $G^{\prime}$ is planar, because a plane embedding of $G^{\prime}$ can be obtained from a plane embedding of $G$ by drawing edge $x_{i} x_{j}$ in a face of $G$ that contains the 2 -walk $x_{i} w x_{j}$. Moreover, $G^{\prime}$ satisfies the restrictions $(a)$ and (b). Since $x_{i}, x_{j}$ have a 2-neighbor, each of them is of degree $\geq 12$ in $G$, which implies that $G^{\prime}$ has no light edge. Finally observe that conditions $\operatorname{deg}\left(x_{i}\right) \geq 12$ and $\operatorname{deg}\left(x_{j}\right) \geq 12$ imply that no crown contains the new edge $x_{i} x_{j}$, and consequently $G^{\prime}$ contains no crown. Hence, $G^{\prime}$ contradicts the maximality of $G$. This establishes our auxiliary claim, that $i=j \pm 1$ $(\bmod k)$.

Since each of $x_{0} x_{1}$ and $x_{2} x_{3}$ has weight at least 14, it easily follows that $\operatorname{deg}\left(x_{0}\right)+$ $\operatorname{deg}\left(x_{2}\right) \geq 14$ or $\operatorname{deg}\left(x_{1}\right)+\operatorname{deg}\left(x_{3}\right) \geq 14$; say the latter holds. Consider the graph $G+x_{1} x_{3}$, where $x_{1} x_{3}$ is inserted in $f$. The above auxiliary claim implies that $x_{1}$ and $x_{3}$ belong to 
a common face in $G$, hence the resulting graph is planar. Again, one can show that this graph contradicts the maximality of $G$. This establishes Claim 1.

Note that the above claim implies that $G$ has no bridges, and so the length of a face is the same as the number of (distinct) edges incident with it. Claim 1 and the fact that there are no 1-crowns in $G$ easily imply the following:

Claim 2. Every face $f$ of $G$ is of length $\ell(f)=3,4,5$ or 6 . Moreover, for $\ell(f)=4,5,6$ face $f$ is incident with $\ell(f)-3$ vertices of degree 2 .

Initial charge. Let $F(G)$ denote the set of faces of $G$. We assign a charge to each vertex and face of $G$. For every $x \in V(G)$, we define the initial charge $c(x)=\operatorname{deg}(x)-4$. Similarly, for every $f \in F(G)$, let $c(f)=\ell(f)-4$. By Euler's formula the total sum of charge assigned to vertices and faces is

$$
\begin{array}{r}
\sum_{x \in V(G) \cup F(G)} c(x)=\sum_{v \in V(G)}(\operatorname{deg}(v)-4)+\sum_{f \in F(G)}(\ell(f)-4)= \\
=2|E(G)|-4|V(G)|+2|E(G)|-4|F(G)|=-8 .
\end{array}
$$

Notice that only 2-vertices, 3-vertices and 3-faces have negative initial charge. Our goal is to redistribute charge between vertices and faces according to prescribed rules in such a way that the total sum of charge will be nonnegative, which will contradict (1). This contradiction will settle the theorem.

Rules. We use the following discharging rules to redistribute charge between vertices and faces.

(R1) A 2-vertex receives 1 unit from each of its two neighbors.

(R2) A 3-vertex receives $1 / 3$ of a unit from each of its three neighbors.

(R3) A 3-face $v_{1} v_{2} v_{3}$ with $\operatorname{deg}\left(v_{1}\right) \leq 5$, receives $1 / 2$ of a unit from each of $v_{2}$ and $v_{3}$.

Let $f$ be a face and let $v_{1}, v_{2}, v_{3}$ be three consecutive vertices incident with $f$ such that $\operatorname{deg}\left(v_{2}\right) \geq 6$.

(R4) If both $v_{1}$ and $v_{3}$ are of degree $\geq 6$ then $v_{2}$ sends $1 / 3$ of a unit to $f$.

(R5) If $\ell(f) \geq 4$, one of $v_{1}, v_{3}$ is of degree 2 and the other is of degree $\geq 6$, then $v_{2}$ receives $1 / 6$ from $f$.

(R6) If $\ell(f) \geq 4$, and both of $v_{1}, v_{3}$ are of degree 2 then $v_{2}$ receives $2 / 3$ from $f$.

Since we deal with multigraphs, the multiple incidence/adjacency is considered in the application of these rules. Thus, for example, if a 3 -vertex $x$ is adjacent to a vertex $v$ by two edges, then $v$ sends the amount $\frac{1}{3}+\frac{1}{3}$ of a unit of charge to $x$ by (R2). 
Final Charge. Here we will prove that for each $x \in V(G) \cup F(G)$, the final charge $c^{*}(x)$ is non-negative, i.e. $c^{*}(x) \geq 0$. Let $f$ be an arbitrary face of $G$. By Claim 2, $\ell(f) \in\{3,4,5,6\}$. Hence we consider four cases:

$\ell(f)=3$ : If $f$ contains a vertex of degree at most 5 , then $c^{*}(f)=0$ by (R3). Otherwise, all three neighbors are of degree $\geq 6$, so it gets $1 / 3$ from each of them by (R4). Hence, $c^{*}(f)=0$.

$\ell(f)=4$ : In this case, by Claim $2, f$ contains exactly one 2-vertex. Let $f=x_{1} x_{2} x_{3} x_{4}$ with $\operatorname{deg}\left(x_{4}\right)=2$. If $\operatorname{deg}\left(x_{2}\right) \leq 5$ then $f$ sends no charge, and so $c(f)=c^{*}(f)=0$. If $\operatorname{deg}\left(x_{2}\right) \geq 6, f$ gets $1 / 3$ from $x_{2}$ by (R4), and it sends $1 / 6$ to each of $x_{1}$ and $x_{3}$ by (R5). This yields $c^{*}(f)=0$.

$\ell(f)=5$ : By Claim 2, $f$ contains exactly two 2-vertices, and so we can assume that $f=$ $x_{1} x_{2} x_{3} x_{4} x_{5}$ with $\operatorname{deg}\left(x_{1}\right)=\operatorname{deg}\left(x_{3}\right)=2$. Then $f$ sends $1 / 6$ to each of $x_{4}, x_{5}$ by (R5), and it sends $2 / 3$ to $x_{2}$ by (R6). Hence, $c^{*}(f)=0$.

$\ell(f)=6$ : By Claim 2, $f$ has three 2-vertices alternating with three vertices of degree at least 12 . Each of the latter receives $2 / 3$ by (R6), which implies that the final charge of $f$ is 0 .

We consider now the final charge of the vertices. By rules (R1) and (R2), it is obvious that 2- and 3-vertices have non-negative final charge, and 4- and 5-vertices do not alter their charge, which is non-negative.

Suppose now that a vertex $v$ is of degree $d \in\{6,7,8\}$. Then, it may send charge only to incident faces by rule (R4). Moreover, if some incident face is a triangle then its two other vertices have degrees at least 6 , which implies that each such triangle receives $1 / 3$ from $v$. Hence,

$$
c^{*}(v) \geq d-4-\frac{d}{3} \geq 0 .
$$

Next suppose that $v$ is of degree $d \in\{9,10\}$. It may send charge only to incident faces by rules (R3) and (R4) and each such face receives at most $1 / 2$ from $v$. Hence,

$$
c^{*}(v) \geq d-4-\frac{d}{2} \geq 0
$$

Suppose now that $v$ is of degree 11 . Notice that $v$ is not adjacent to a 2 -vertex, and so it sends charge to a neighbor only if it is a 3-vertex. Since by Claim 2, no two 3-neighbors of $v$ are consecutive in clockwise order around $v$, the number of 3-neighbors is at most 5 . Notice that $v$ sends $1 / 2$ to at most 10 faces, and to the remaining faces it sends at most $1 / 3$. Hence,

$$
c^{*}(v) \geq 7-\frac{10}{2}-\frac{1}{3}-\frac{1}{3} \cdot 5=0 .
$$

Finally suppose that $d \geq 12$. Let $x_{0}, x_{1}, \ldots, x_{d-1}$ be the neighbors of $v$ enumerated in clockwise order around $v$, and let $f_{i}$ be the face incident with the walk $x_{i} v x_{i+1}$ (throughout 
this proof we take the indices in $x_{i}$ modulo $d$ ). We consider a few cases regarding the number $d_{2}$ of 2 -vertices adjacent to $v$.

Case 1: $d_{2}=0$. Since $v$ sends at most $1 / 2$ to each incident face and it has at most $\left\lfloor\frac{d}{2}\right\rfloor$ adjacent 3-neighbors, its final charge is

$$
c^{*}(v) \geq d-4-\frac{d}{2}-\frac{1}{3}\left\lfloor\frac{d}{2}\right\rfloor \geq \frac{d}{3}-4 \geq 0 .
$$

Case 2: $d_{2}=1$. Let $x_{1}$ be the 2-neighbor of $v$. By Claim 1, without loss of generality we may assume that $f_{0}$ is a 3 -face and $f_{1}$ is a face of length 4 or $5\left(f_{1}\right.$ cannot be a face of length 6 since then $f_{1}$ contains two 2-neighbors of $v$ so $d_{2} \geq 2$ ). Notice that $v$ sends 1 to $x_{1}$ and $1 / 2$ to $f_{0}$. Next, it sends nothing to $f_{1}$ and $\leq 1 / 2$ to each of the $d-2$ remaining faces. Finally, it sends at most $\frac{1}{3}\left\lfloor\frac{d-1}{2}\right\rfloor$ to its adjacent 3 -vertices. If $d \geq 13$, then

$$
c^{*}(v) \geq d-4-1-\frac{1}{2}-\frac{d-2}{2}-\frac{1}{3}\left\lfloor\frac{d-1}{2}\right\rfloor \geq 0 .
$$

Now assume that $d=12$. We consider two subcases regarding the degree of $x_{2}$. If $\operatorname{deg}\left(x_{2}\right) \geq 6$, then $f_{1}$ sends $\frac{1}{6}$ to $v$ by (R5), and we conclude

$$
c^{*}(v) \geq d-4-1-\frac{1}{2}-\frac{d-2}{2}-\frac{1}{3}\left\lfloor\frac{d-1}{2}\right\rfloor+\frac{1}{6}=0 .
$$

Finally, since $d$ is even, if $\operatorname{deg}\left(x_{2}\right) \leq 5$ then there is a face distinct from $f_{1}$ that receives at most $1 / 3$ from $v$. In that case, we obtain

$$
c^{*}(v) \geq d-4-1-\frac{1}{2}-\frac{d-3}{2}-\frac{1}{3}-\frac{1}{3}\left\lfloor\frac{d-1}{2}\right\rfloor=0 .
$$

Case 3: $d_{2} \geq 2$. Observe that since the rules move charge only between incident faces and vertices, while calculating the charge sent by $v$ we can restrict ourselves only to $v$ and its adjacent vertices and incident faces. In order to make the argument shorter, we use the following claim:

Claim 3. We can modify the neighborhood of $v$ so that every 2-vertex $x_{i}$ is adjacent to $x_{i-1}$ and the final charge $c^{*}(v)$ stays the same.

Let $\operatorname{deg}_{G}\left(x_{i}\right)=2$. Then by Claim $1, x_{i}$ is adjacent to $x_{i-1}$ or $x_{i+1}$. Assume that it is adjacent to $x_{i+1}$. Then $x_{i+2}$ is not a 2 -vertex adjacent to $x_{i+1}$, since $G$ does not contain a 1 -crown. Then we remove $x_{i}$, and we draw it inside face $f_{i+1}$ together with the edges to $v$ and $x_{i+1}$. In the new drawing, let us rename the vertices and faces so that they are still enumerated in clockwise order. In particular, $x_{i+1}$ is renamed as $x_{i}^{\prime}, x_{i}$ is renamed as $x_{i+1}^{\prime}$, and for every $j \neq i, i+1$, vertex $x_{j}$ is renamed as $x_{j}^{\prime}$. In the new drawing, let $f_{i}^{\prime}$ be the face incident with the walk $x_{i}^{\prime} v x_{i+1}^{\prime}$. Let $c_{j}$ (respectively $c_{j}^{\prime}$ ) be the charge sent from $v$ to $f_{j}$ (respectively $f_{j}^{\prime}$ ) minus the charge received by $v$ from $f_{j}$ (respectively $f_{j}^{\prime}$ ). 
Obviously the charge sent/received by $v$ to/from neighbors of $v$ has not changed. Also, $c_{j}^{\prime}=c_{j}$, for $j \neq i-1, i+1$. If $\operatorname{deg}_{G}\left(x_{i-1}\right)=2$ then by Claim $2, f_{i-1}$ is of length 5 or 6 , so by (R5) and (R6), $c_{i-1}^{\prime}-c_{i-1}=-1 / 6-(-2 / 3)=1 / 2$. If $\operatorname{deg}_{G}\left(x_{i-1}\right)=3,4,5$ then there is no 2 -vertex adjacent to $x_{i-1}$, so $f_{i-1}$ is a 4 -face and $f_{i-1}^{\prime}$ is a 3 -face, hence by (R3), $c_{i-1}^{\prime}-c_{i-1}=1 / 2$. Finally, when $\operatorname{deg}_{G}\left(x_{i-1}\right) \geq 6$ then $f_{i-1}$ is of length 4 or 5 , so by (R4) and (R5), $c_{i-1}^{\prime}-c_{i-1}=1 / 3-(-1 / 6)=1 / 2$. Hence $c_{i-1}^{\prime}-c_{i-1}=1 / 2$ in all cases. Analogously one can verify that no matter what is the degree of $x_{i+2}, c_{i+1}^{\prime}-c_{i+1}=-1 / 2$. Hence the charge sent from $v$ remains the same. This settles the claim.

We modify the neighborhood of $v$ as described in Claim 3. Note that if $x_{i}$ is a 2-vertex, then its neighbor $x_{i-1}$ is of degree $\geq 12$. Obviously, this redrawing in Claim 3 introduces neither a crown nor a pair of consecutive $v$ neighbors of $v$ of degree 3,4 , or 5 . Also, $G^{*}$ stays unchanged.

In what follows, we will bound the amount of charge sent by $v$ to faces. Denote by $d_{4,5}$ the number of 4- and 5-neighbors of $v$. Denote by $f_{-1 / 6}$ and $f_{1 / 3}$ the number of faces which send $1 / 6$ to $v$ or receive $1 / 3$ from $v$, respectively. Let $x_{i}$ and $x_{j}$ be two distinct 2-neighbors of $v$, such that for each $k \in\{i+1, \ldots, j-1\}, \operatorname{deg}\left(x_{k}\right)>2$. If there is a crown whose vertices belong to $\left\{v, x_{i-1}, x_{i}, x_{i+1}, \ldots, x_{j}\right\}$ we call the (ordered) pair $\left(x_{i}, x_{j}\right)$ bad, otherwise it is good. Let $b$ denote the number of bad pairs. Note that there are $d_{2}-b$ good pairs.

Claim 4. For any good pair $\left(x_{i}, x_{j}\right)$ one of the following conditions holds:

(A) $\operatorname{deg}_{G}\left(x_{i+1}\right) \geq 6$,

(B) for some $k \in\{i+1, \ldots, j-2\} \operatorname{deg}\left(x_{k}\right) \geq 6$ and $\operatorname{deg}\left(x_{k+1}\right) \geq 6$,

(C) for some $k \in\{i+1, \ldots, j-2\}, \operatorname{deg}_{G}\left(x_{k}\right) \in\{4,5\}$.

Assume that none of the above conditions holds. Note that by Claim $3, j \neq i+1$. Then, the following property holds: for each $k \in\{i+1, \ldots, j-1\}, \operatorname{deg}_{G}\left(x_{k}\right) \geq 6$ if $k$ has the same parity as $i$ and $\operatorname{deg}_{G}\left(x_{k}\right)=3$ otherwise. Let $H$ be the subgraph of $G$ with $V(H)=\left\{v, x_{i-1}, x_{i}, x_{i+1}, \ldots, x_{j}\right\}$ and $E(H)=\left\{v x_{k}: k \in\{i-1, i, \ldots, j\}\right\} \cup$ $\left\{x_{i-1} x_{i}, x_{i-1} x_{i+1}\right\} \cup\left\{x_{k} x_{k+1}: k \in\{i+1, \ldots, j-1\}\right\}$. Then $H$ is a crown around $v$, unless some pair of its vertices $x_{a}, x_{b}$ coincide. Notice that then $\operatorname{deg}\left(x_{a}\right)=\operatorname{deg}\left(x_{b}\right) \geq 6$. As long as there is such a pair in $H$ we remove from $H$ all the vertices and edges inside the 2-cycle $v x_{a} x_{b}$ and we remove edge $v x_{b}$. The resulting subgraph $H$ is a crown around $v$ with vertices in the set $\left\{v, x_{i-1}, x_{i}, x_{i+1}, \ldots, x_{j}\right\}$, which is a contradiction. This settles the claim.

Observe that in case (A) face $f_{i}$ sends $1 / 6$ to $v$ by (R5), and in case (B) face $f_{k}$ receives precisely $1 / 3$ from $v$ by (R4). As there are $d_{2}-b$ good pairs it follows that $f_{-1 / 6}+f_{1 / 3}+d_{4,5} \geq d_{2}-b$. Thus, some $d_{2}-b-f_{-1 / 6}-d_{4,5}$ faces receive precisely $1 / 3$ from $v$. Note that for any 2-vertex $x_{i}$, the face $f_{i}$ does not receive a charge from $v$. Thus, there are $d_{2}$ faces which do not receive any charge from $v$. Each of the remaining 
$d-d_{2}-\left(d_{2}-b-f_{-1 / 6}-d_{4,5}\right)$ faces receives at most $1 / 2$ unit from $v$. Now we bound the total charge sent from $v$ to faces minus the charge received from faces. It amounts at most:

$$
\begin{array}{r}
\frac{1}{3}\left(d_{2}-b-f_{-1 / 6}-d_{4,5}\right)+\frac{1}{2}\left[d-d_{2}-\left(d_{2}-b-\right.\right. \\
\left.\left.f_{-1 / 6}-d_{4,5}\right)\right]-\frac{1}{6} f_{-1 / 6} \\
=\frac{d}{2}-\frac{2}{3} d_{2}+\frac{b}{6}+\frac{d_{4,5}}{6} .
\end{array}
$$

In the sequel we estimate the charge $v$ sends to neighbors. We start from bounding the number of 3-neighbors of $v$. Consider (cyclically) the degree sequence $S_{0}=\operatorname{deg}\left(x_{0}\right)$, $\operatorname{deg}\left(x_{1}\right), \ldots, \operatorname{deg}\left(x_{d-1}\right)$. First remove elements with value 2 from this sequence. If two consecutive elements of the resulting sequence $S_{1}$ have value each at least 6 , we will call them a big pair. Observe that if (A) holds in Claim 4, then by Claim $3 \operatorname{deg}_{G}\left(x_{i-1}\right) \geq 12$ and consequently $\operatorname{deg}_{G}\left(x_{i-1}\right)$ and $\operatorname{deg}_{G}\left(x_{i+1}\right)$ are a big pair. Hence by Claim 4 , in $S_{1}$ there are at least $\left(d_{2}-b\right)-d_{4,5}$ big pairs (we consider the last element of $S_{1}$ to be consecutive with the first one). Next, as long as the sequence contains a big pair we remove one of the elements of the pair, unless the sequence consists of only two elements, each of value at least 6 . In the latter case both these elements are removed. After these two steps, the resulting sequence $S_{2}$ has length $\leq d-d_{2}-\left(d_{2}-b-d_{4,5}\right)$. By Claim 1 , and because edges have weight at least 14 , it follows that in $G^{*}$ vertex $v$ has no pair of consecutive neighbors both of degree 3,4 or 5 . It follows that sequence $S_{2}$ does not contain a pair of consecutive elements equal to 3,4 or 5 . Thus, $S_{2}$ contains at most $\left\lfloor\frac{d-d_{2}-\left(d_{2}-b-d_{4,5}\right)}{2}\right\rfloor=\left\lfloor\frac{d+b+d_{4,5}}{2}\right\rfloor-d_{2}$ elements equal to 3, 4 or 5, and hence this is an upper bound for the number of 3-, 4and 5-neighbors of $v$. It follows that $v$ has at most $\left\lfloor\frac{d+b+d_{4,5}}{2}\right\rfloor-d_{2}-d_{4,5}=\left\lfloor\frac{d+b-d_{4,5}}{2}\right\rfloor-d_{2}$ neighbors of degree 3 . Thus, the total charge sent from $v$ to its neighbors is at most

$$
d_{2}+\frac{1}{3}\left(\left\lfloor\frac{d+b-d_{4,5}}{2}\right\rfloor-d_{2}\right) .
$$

Finally, by (2) and (3) we conclude that

$$
\begin{aligned}
c^{*}(v) & \geq d-4-d_{2}-\frac{1}{3}\left(\left\lfloor\frac{d+b-d_{4,5}}{2}\right\rfloor-d_{2}\right)-\left(\frac{d}{2}-\frac{2}{3} d_{2}+\frac{b}{6}+\frac{d_{4,5}}{6}\right) \\
& \geq \frac{d}{3}-4-\frac{b}{3} .
\end{aligned}
$$

Each $k$-crown contains $k-1$ vertices of degree 3 , which are neighbors of $v$. For each bad pair $\left(x_{i}, x_{j}\right)$ there is a crown with vertices from $\left\{v, x_{i-1}, \ldots, x_{j}\right\}$. Since small crowns are excluded, such a crown contains at least five 3-neighbors of $v$. Hence $v$ has at least $5 b$ neighbors of degree 3. By Claim 1, each 3-neighbor of $v$ is incident in $G^{*}$ with two triangular faces containing $v$. Each of these faces contains also a neighbor of $v$ of degree at least 11, as light edges are excluded. The edge joining $v$ and its neighbor can belong to at most 2 of these faces. Consequently there are at least $5 b$ edges joining $v$ and its neighbors of degree at least 11 . Finally, $v$ has at least $b$ neighbors of degree 2 . It follows that $\operatorname{deg}_{G}(v) \geq 11 b$ and so $b \leq\left\lfloor\frac{d}{11}\right\rfloor$. 
Hence for $d \geq 14$, we get $c^{*}(v) \geq \frac{d}{3}-4-\frac{1}{3} \cdot \frac{d}{11}>0$. For $d=13$, we get $c^{*}(v) \geq$ $\frac{d}{3}-4-\frac{1}{3}=0$. Observe that Claim 1 implies that all the vertices of a crown around $v$, except for $v$, are adjacent to $v$. Hence a crown around $v$ implies that at least 13 edges are incident with $v$, for it has size at least 6 . Consequently, for $d=12$, there are no crowns around $v$ and $c^{*}(v) \geq \frac{d}{3}-4=0$.

This completes the case $d \geq 12$. We infer that every vertex and face has non-negative charge after the rules are applied, which is a contradiction. This establishes the proof.

In Theorem 1.5 the number 5 is best possible in the sense that there is a planar graph with minimum degree 2 with no crowns of size smaller than 5 and with no light edges. To construct such a graph take a triangulation $T$ with vertices of degree 5 and 6 such that 5 -vertices are at distance at least 5 from each other; for example the duals of some fullerens are such graphs. Then, for each 5 -vertex $x$ of $T$ we choose one incident triangle and we remove its edge not incident with $x$. As a result we get a graph $T^{\prime}$ with faces of length 3 and 4. Next, we put a vertex into each face of $T^{\prime}$ and we join it with the vertices incident with the face. Denote the resulting triangulation by $T^{\prime \prime}$. Observe that every light edge in $T^{\prime \prime}$ joins a 3 -vertex with a 10-vertex. Moreover, the 10 -vertex is adjacent to a 4 -vertex. For each 4-vertex $y \in V\left(T^{\prime \prime}\right)$ let its neighbors be $y_{0}, y_{1}, y_{2}, y_{3}$ in clockwise order. Finally, for each $i \in\{0,1,2,3\}$ we add a new 2 -vertex connected to $y_{i}$ and $y_{i+1}$ (indices modulo 4). Clearly, the resulting graph $G$ has vertices of degree 2, 3, 12, and 14 only. Vertices of degree 2 and 3 are adjacent to vertices of degree 12 or 14 . Hence there are no light edges in $G$. One may verify that $G$ contains crowns of size 5 and 6 but no crowns of smaller size.

\section{$3 \quad$ Reducibility of Crowns}

In this section we show that crowns are reducible. Although we use crowns of size at most 5 , here we consider all crowns. In the next lemma we will use the well-known fact that every even cycle is 2-edge-choosable.

Lemma 3.1. Let $G$ be a graph of maximum degree $\Delta$ and let $S$ be a $k$-crown in $G, k \geq 1$. Let $L$ be a list assignment of $G$ such that $|L(e)| \geq \Delta$ for every edge $e \in E(G)$. Then any $L$-coloring of $G-E(S)$ can be extended to an $L$-coloring of $G$.

Proof. Let $\lambda$ be an arbitrary $L$-edge-coloring of $G-E(S)$. For every $e \in E(S)$, let $I(e)$ denote the set of edges from $E(G)-E(S)$ that are incident with $e$ and let $L^{\prime}(e)=$ $L(e) \backslash \bigcup_{f \in I(e)} \lambda(f)$. Let us denote the vertices of $S$ as in Fig. 1. Recall that $\operatorname{deg}_{G}\left(x_{1}\right)=$ $\operatorname{deg}_{G}\left(x_{2 k+1}\right)=2$ and for every $i=3,5, \ldots, 2 k-1, \operatorname{deg}_{G}\left(x_{i}\right)=3$. Note that for $i=$ $1,3, \ldots, 2 k+1,\left|L^{\prime}\left(v x_{i}\right)\right| \geq k+1$ and for $i=1,2, \ldots, 2 k,\left|L^{\prime}\left(x_{i} x_{i+1}\right)\right| \geq 2$. Without loss of generality we may assume that for $i=1,3, \ldots, 2 k+1,\left|L^{\prime}\left(v x_{i}\right)\right|=k+1$ and for $i=1,2, \ldots, 2 k,\left|L^{\prime}\left(x_{i} x_{i+1}\right)\right|=2$. Clearly in order to extend $\lambda$ to an $L$-coloring of $G$ it suffices to $L^{\prime}$-color the graph $S$. Thus our objective will be to construct an $L^{\prime}$-coloring of $S$, where $L^{\prime}$ is any list assignment with the above prescribed lengths of lists. We do it by induction on $k$. For $k=1$ we must 2-list-color a 4-cycle but even-length cycles are 2-choosable [8, 14]. 
Now, we consider the case $k=2$. We may assume that $L^{\prime}\left(v x_{3}\right) \subseteq L^{\prime}\left(x_{2} x_{3}\right) \cup L^{\prime}\left(x_{3} x_{4}\right)$, for otherwise we color $v x_{3}$ with a color from $L^{\prime}\left(v x_{3}\right) \backslash\left[L^{\prime}\left(x_{2} x_{3}\right) \cup L^{\prime}\left(x_{3} x_{4}\right)\right]$ and then we are left with the problem of 2-list-coloring of a 6-cycle. Since $\left|L^{\prime}\left(v x_{3}\right)\right|=3$, it follows that $L^{\prime}\left(x_{2} x_{3}\right) \neq L^{\prime}\left(x_{3} x_{4}\right)$. Then we color $x_{2} x_{3}$ with a color not in $L^{\prime}\left(x_{3} x_{4}\right)$ and we color $x_{1} x_{2}$ with a free color. We assume now that $v x_{3}$ has two free colors, otherwise we remove one. We may also assume that $v x_{3}$ and $x_{4} x_{5}$ do not have a common free color, for otherwise we color them both with such a color and then we can color $v x_{1}, v x_{5}, x_{3} x_{4}$, in this order, always using a free color. Since $v x_{5}$ has three free colors and both $v x_{3}, x_{4} x_{5}$ have two free colors, either $v x_{3}$ or $x_{4} x_{5}$ has a free color $p \notin L^{\prime}\left(v x_{5}\right)$. In the case $p \in L^{\prime}\left(v x_{3}\right)$ we color $v x_{3}$ with $p$ and then we color the remaining edges in the following order: $v x_{1}, x_{3} x_{4}, x_{4} x_{5}, v x_{5}$. In the latter case we assign color $p$ to $x_{4} x_{5}$ and we color $x_{3} x_{4}, v x_{3}, v x_{1}, v x_{5}$, in this order, always using a free color. This settles the case $k=2$.

Now assume $k \geq 3$. We consider two possibilities:

Case 1: $L^{\prime}\left(x_{2} x_{3}\right)=L^{\prime}\left(x_{3} x_{4}\right)$. Let $r$ be a color from $L^{\prime}\left(v x_{3}\right) \backslash L^{\prime}\left(x_{2} x_{3}\right)$. We remove $x_{3}$ and identify $x_{2}$ with $x_{4}$. For each $i=1,3,4, \ldots, k+1$ let $L^{\prime \prime}\left(v x_{2 i-1}\right)=L^{\prime}\left(v x_{2 i-1}\right) \backslash$ $\{r\}$. The resulting graph is a $(k-1)$-crown, and it is $L^{\prime \prime}$-colorable by the induction hypothesis. Let $\lambda^{\prime \prime}$ be such a coloring. We extend $\lambda^{\prime \prime}$ to an $L^{\prime}$-coloring of $S$ as follows. Let $p \in L^{\prime}\left(x_{2} x_{3}\right) \backslash\left\{\lambda^{\prime \prime}\left(x_{1} x_{2}\right)\right\}$ and $q \in L^{\prime}\left(x_{3} x_{4}\right) \backslash\left\{\lambda^{\prime \prime}\left(x_{4} x_{5}\right)\right\}$. Since $L^{\prime}\left(x_{2} x_{3}\right)=$ $L^{\prime}\left(x_{3} x_{4}\right)$ and $\lambda^{\prime \prime}\left(x_{1} x_{2}\right) \neq \lambda^{\prime \prime}\left(x_{4} x_{5}\right)$, it follows that $p \neq q$. Hence we can color $x_{2} x_{3}$ with $p, x_{3} x_{4}$ with $q$, and $v x_{3}$ with $r$.

Case 2: $L^{\prime}\left(x_{2} x_{3}\right) \neq L^{\prime}\left(x_{3} x_{4}\right)$. Let $L^{\prime}\left(x_{2} x_{3}\right)=\{a, b\}$ and $c \in L^{\prime}\left(x_{3} x_{4}\right), c \notin\{a, b\}$. Then we color $v x_{3}$ with a color distinct from $a, b$, and $c$. This is possible since $\left|L^{\prime}\left(v x_{3}\right)\right|=$ $k+1 \geq 4$. Next, we color $x_{3} x_{4}$ with $c$ and we color $x_{4} x_{5}, x_{5} x_{6}, \ldots, x_{2 k} x_{2 k+1}$, in this order, always using a free color. Now for every $i=5,7, \ldots, 2 k-1, v x_{i}$ has at least $k-2$ free colors and $v x_{2 k+1}$ has at least $k-1$ free colors. Hence, we may color them greedily, i.e., in the order $v x_{5}, v x_{7}, \ldots, v x_{2 k+1}$ always using a free color. Afterwards $v x_{1}$ has at least one free color and both $x_{1} x_{2}, x_{2} x_{3}$ have two free colors, so we color them greedily as well.

Theorem 1.5 and Lemma 3.1 imply the following corollary.

Corollary 3.2. Every planar graph with maximum degree $\Delta \geq 12$ is $\Delta$-edge-choosable.

\section{List-Edge-Coloring Algorithm}

In this section we describe a linear-time algorithm which for a given simple planar graph $G$ and an edge-list assignment $L$, computes an $L$-edge-coloring of $G$, provided that for every $e \in E(G),|L(e)|=\max \{\Delta(G), 12\}$. The algorithm does not need a plane embedding of graph $G$. In fact, one can use the algorithm for any class of graphs which can replace planar graphs in Theorem 1.5. 
We assume that the input graph $G$ is given in the form of adjacency lists. Also the list assignment is stored as an array of lists, one list for each edge. Additionally, we assume that each list of admissible colors is sorted. Equivalently, one can assume that the largest color has value $\mathcal{O}(|E(G)| \Delta)$. Then the lists can be sorted in linear time using bucket-sort.

In the following subsection we describe some tools used by our coloring algorithm. Then we describe the main body of the algorithm and we analyze its time complexity.

\subsection{Efficient Coloring and Finding Small Crowns}

Lemma 4.1. Let $G$ be a graph of maximum degree $\Delta$ containing an edge e of weight at most $\max \{\Delta+1,13\}$. Let $L$ be an edge-list assignment of $G$ such that $|L(e)| \geq \max \{\Delta, 12\}$ for every edge $e \in E(G)$. Then any $L$-edge-coloring of $G-\{e\}$ can be extended to an $L$ edge-coloring of $G$ in $\mathcal{O}(\Delta)$ time.

Proof. Let $\lambda$ denote the $L$-edge-coloring of $G-\{e\}$ and let $I(e)$ denote the set of edges incident with $e$ and let $L^{\prime}(e)=L(e) \backslash \bigcup_{f \in I(e)} \lambda(f)$. Clearly $\left|L^{\prime}(e)\right| \geq 1$. The algorithm simply colors $e$ with any color from $L^{\prime}(e)$. In order to find $L^{\prime}(e)$ efficiently, each vertex $x$ in graph $G$ stores a sorted list Used $(x)$ of colors used by the already colored incident edges. As the list $L(e)$ is also sorted, the set $L^{\prime}(e)$ can be easily found in $\mathcal{O}(\Delta)$ time. Additionally, after coloring the edge $e=x y$, both lists Used $(x)$ and Used $(y)$ are updated in $\mathcal{O}(\Delta)$ time.

The following lemma states that the proof of Lemma 3.1 can be transformed into an efficient algorithm when $k=\mathcal{O}(1)$.

Lemma 4.2. Let $G$ be a graph of maximum degree $\Delta$ and let $S$ be a k-crown in $G$, $k=\mathcal{O}(1)$. Let $L$ be an edge-list assignment of $G$ such that $|L(e)| \geq \Delta$ for every edge $e \in E(G)$. Then any L-edge-coloring of $G-E(S)$ can be extended to an L-edge-coloring of $G$ in $\mathcal{O}(\Delta)$ time.

Proof. We consider the algorithm arising from the proof of Lemma 3.1. Each of the sets $L^{\prime}(e)$ from the proof of Lemma 3.1 is computed in $\mathcal{O}(\Delta)$ time, as described in the proof of Lemma 4.1. As $k=\mathcal{O}(1)$, this whole phase takes $\mathcal{O}(\Delta)$ time. Afterwards, we deal with bounded-sized graphs and bounded-sized list assignments hence the remaining part of coloring algorithm takes constant time. Finally, as in the proof of Lemma 4.1 relevant sets Used $(\cdot)$ are updated in $\mathcal{O}(\Delta)$ time.

Now we consider algorithm $\operatorname{SearchSmallCrown}(G, x)$ (see Alg. 4.1) which will be used for searching for small crowns.

Lemma 4.3. Let $x$ and $v$ be distinct vertices in a graph $G$ and let $\operatorname{deg}_{G}(x) \in\{2,3\}$. Assume that in $G$ there is a small crown around $v$ containing $x$. Then algorithm SearchSmallCrown $(G, x)$ returns a light edge or the edges of a small crown. Moreover, its time complexity is $\mathcal{O}(\Delta)$. 


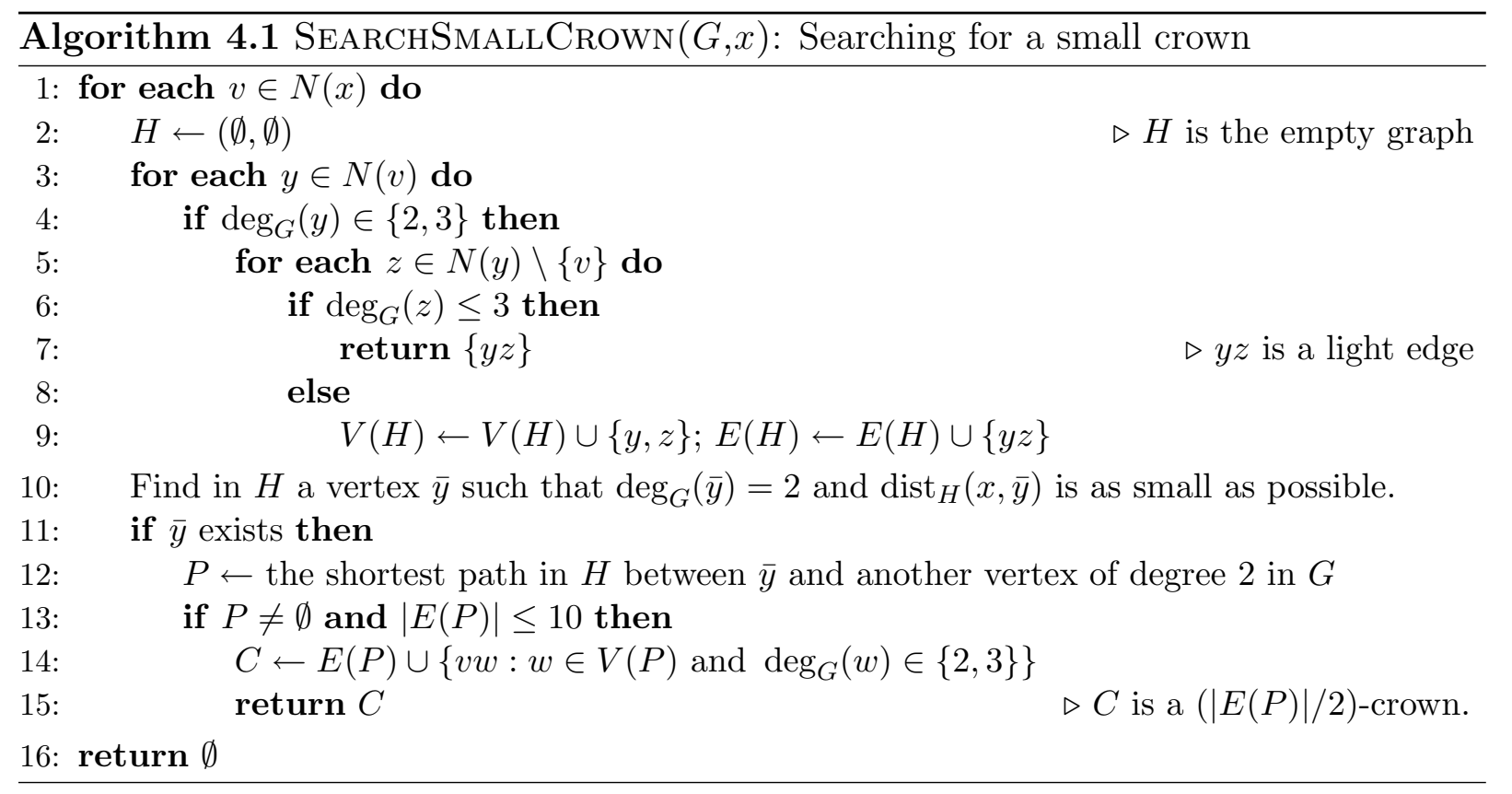

Proof. First assume that the algorithm returns set $C$ in line 15 . We will show that $C$ contains the edges of a small crown. Since a light edge was not returned in line 7 , then for some vertex $v$, which is a neighbor of $x$,

$$
E(H)=\left\{y z: y \in N(v), \operatorname{deg}_{G}(y) \in\{2,3\}, z \in N(y)-\{v\} \text { and } \operatorname{deg}_{G}(z)>3\right\} .
$$

Note that $H$ is a bipartite graph with partite sets $Y=\left\{y \in V(H): \operatorname{deg}_{G}(y) \in\{2,3\}\right\}$ and $Z=\left\{z \in V(H): \operatorname{deg}_{G}(z)>3\right\}$. Hence $P$ has even length, as both its ends have degree 2 in $G$. Let $y_{0}, z_{1}, y_{1}, z_{2}, y_{2}, \ldots, z_{|E(P)| / 2}, y_{|E(P)| / 2}$ be the successive vertices of $P$. Note that these vertices are all distinct for otherwise $P$ is not the shortest path in $H$ between $\bar{y}$ and another 2-vertex. By (4) each vertex $y_{i}$ of path $P$ is adjacent to $v$. Note that $P$ contains at least two edges, since it has distinct ends. It follows that $C$ contains edges of a crown around $v$ of size $|E(P)| / 2 \leq 5$.

Now it suffices to show that the algorithm returns a light edge in line 7 or returns set $C$ in line 15. Assume that neither of these happens. Let $S$ be a small crown around $v$ containing $x, v \neq x$. ( $S$ exists by the assumptions of the lemma). Let $k$ denote the size of $S$. In lines 2 to 9 the algorithm finds the subgraph $H \subseteq G$ with edge set described in (4). Let $x_{1}, x_{2}$ be the neighbors of $v$ in $S$ with degree 2 in $G$. Observe that $E(S-v) \subseteq E(H)$. In line 10 the algorithm finds some vertex $\bar{y}$, because $S$ contains $x, x_{1}$ and $x_{2}$ (possibly $x=x_{1}$ or $x=x_{2}$ ). If $\bar{y}=x_{1}$ (respectively $\bar{y}=x_{2}$ ) then there is a path in $H$ from $\bar{y}$ to another vertex of degree 2 in $G$, namely $x_{2}$ (respectively $x_{1}$ ). Consequently when $\bar{y} \in\left\{x_{1}, x_{2}\right\}$ the algorithm finds some path $P$ in line 12 , and $|E(P)| \leq 2 k$. Also if $\bar{y} \notin\left\{x_{1}, x_{2}\right\}$ then $H$ contains a path from $\bar{y}$ to $x$ and a path from $x$ to $x_{1}$, hence some path $P$ is found. 
Moreover, then $\operatorname{dist}_{H}(x, \bar{y}) \leq \min \left\{\operatorname{dist}_{H}\left(x, x_{1}\right), \operatorname{dist}_{H}\left(x, x_{2}\right)\right\}$ and so

$$
\begin{aligned}
|E(P)| & \leq \operatorname{dist}_{H}(\bar{y}, x)+\min \left\{\operatorname{dist}_{H}\left(x, x_{1}\right), \operatorname{dist}_{H}\left(x, x_{2}\right)\right\} \\
& \leq 2 \min \left\{\operatorname{dist}_{H}\left(x, x_{1}\right), \operatorname{dist}_{H}\left(x, x_{2}\right)\right\} \\
& \leq 2 k
\end{aligned}
$$

It follows that $|E(P)| \leq 10$. Hence line 15 is executed, a contradiction. This proves that the algorithm returns a small crown or a light edge.

Clearly, graph $H$ has $\mathcal{O}(\Delta)$ size and building it takes $\mathcal{O}(\Delta)$ time. The other part of the algorithm can be easily implemented using Breadth First Search and then it takes time linear respect in the size of $H$, i.e. $\mathcal{O}(\Delta)$ time.

\subsection{Main Body of the Algorithm}

Now we describe algorithm EdGeListColor, which edge-list-colors an input simple planar graph $G$ with edge color lists of length $\max \{\Delta(G), 12\}$. Our algorithm uses a queue $Q$ which stores vertices around which one should look for light edges and small crowns. It is initialized with the set of all vertices of $G$. However, one vertex may appear several times in $Q$.

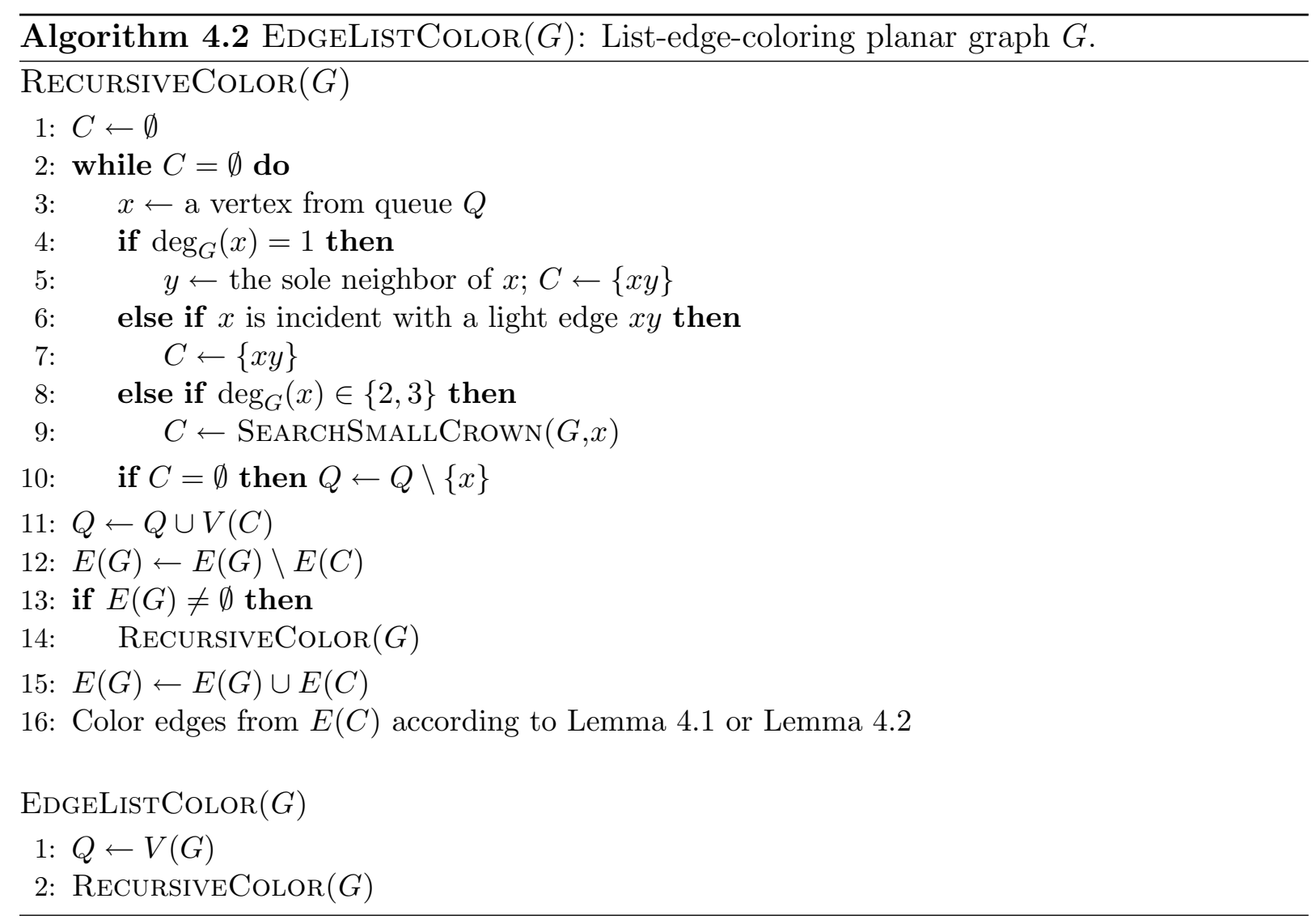


After the initialization the algorithm calls a recursive routine $\operatorname{ReCURSIVECoLOR}(G)$ (see Alg. 4.2). Let us consider one such recursive call. Consider the following assertion:

$Q$ contains all 1-vertices and endpoints of light edges in $G$; for any small crown $C$ around $v$ in $G$ queue $Q$ contains a vertex $x \in V(C) \backslash\{v\}, \operatorname{deg}_{G}(x) \in\{2,3\}$.

Obviously, the assertion holds after initialization. Then, each time some set of edges is removed from the graph, the endpoints of these edges are added to $Q$ in line 11. Also, if a vertex $x$ is removed from $Q$ and not inserted again then $\operatorname{deg}_{G}(x) \neq 1$, there is no light edge incident with $x$ and either $\operatorname{deg}_{G}(x) \notin\{2,3\}$ or there is no small crown containing it. This proves that the assertion always holds at the beginning of the RECURSIVEColor $(G)$ routine. The assertion together with Theorem 1.5 and Lemma 4.3 guarantees that in line 11 set $C$ contains a single edge of weight $\Delta+1$, a single light edge, or the edges of a small crown. This easily implies the following:

Corollary 4.4. Algorithm $\operatorname{EdgeListColor}(G)$ properly colors a planar graph $G$.

Proposition 4.5. The time complexity of algorithm EdGeListColor is $\mathcal{O}(|V(G)| \Delta)$.

Proof. Since in each recursive call at least one edge is removed, there are $\mathcal{O}(|E(G)|)=$ $\mathcal{O}(|V(G)|)$ recursive calls. In each recursive call $\mathcal{O}(1)$ vertices are added to $Q$, hence in total $\mathcal{O}(|V(G)|)$ vertices are added to $Q$. A straightforward implementation of line 6 works in $\mathcal{O}(\Delta)$ time. Line 9 takes $\mathcal{O}(\Delta)$ time by Lemma 4.3. Hence the total time spent on lines $1-10$ is $\mathcal{O}(|V(G)| \Delta)$.

Finally, as the number of recursive calls is $\mathcal{O}(|V(G)|$, by Lemmas 4.1 and 4.2 the total time spent on lines $11-16$ is $\mathcal{O}(|V(G)| \Delta)$. This settles the proof.

\section{Acknowledgments}

The authors thank anonymous referees for reading the paper carefully and for providing many helpful comments.

\section{References}

[1] O. V. Borodin. On the total coloring of planar graphs. J. Reine Ange. Math., (394):180-185, 1989.

[2] O. V. Borodin. Joint extension of two theorems of Kotzig on 3-polytopes. Combinatorica, 13(1):121-125, 1993.

[3] O. V. Borodin, A. V. Kostochka, and D. R. Woodall. List edge and list total colourings of multigraphs. J. Comb. Theory Ser. B, 71:184-204, 1997.

[4] M. Chrobak and T. Nishizeki. Improved edge-coloring algorithms for planar graphs. J. Algorithms, 11:102-116, 1990. 
[5] M. Chrobak and M. Yung. Fast algorithms for edge-coloring planar graphs. J. Algorithms, 10:35-51, 1989.

[6] R. Cole and Ł. Kowalik. New linear-time algortihms for edge-coloring planar graphs. To appear in Algorithmica.

[7] Z. Dvorak and R. Škrekovski. A theorem about a contractible and light edge. SIAM J. Discrete Math., 20(1):55-61, 2006.

[8] P. Erdős, A. L. Rubin, and H. Taylor. Choosability in graphs. Congressus Numerantium, 26:125-157, 1980.

[9] H. Gabow and S. Skulrattanakulchai. Coloring algorithms on subcubic graphs. Internat. J. Found. Comput. Sci., 15(1):21-40, 2004.

[10] S. Jendrol' and H.-J. Voss. Light subgraphs of graphs embedded in 2-dimensional manifolds of Euler characteristic $\leq 0-$ a survey. In G. Halász, L. Lovász, M. Simonovits, and V. Sós, editors, Paul Erdös and His Mathematics II, volume 11 of Bolyai Society Mathematical Studies, pages 375-411. Springer, 2002.

[11] S. Jendrol' and H.-J. Voss. Light subgraphs of graphs embedded in the plane and in the projective plane - a survey. To appear in Discrete Math.

[12] A. Kotzig. Contribution to the theory of eulerian polyhedra. Math. Čas. SAV (Math. Slovaka), (5):111-113, 1955.

[13] B. Mohar, R. Škrekovski, and H.-J. Voss. Light subgraphs in planar graphs of minimum degree 4 and edge-degree 9. J. Graph Theory, 44:261-295, 2003.

[14] V. G. Vizing. Vertex colorings with given colors (in Russian). Metody Diskret. Analiz, 29:3-10, 1976.

[15] J. Zaks. Extending Kotzig's theorem. Israel J. Math., 45(4):281-296, 1983. 